\title{
CONFUSIÓN DE LENGUAS EN LA PAREJA HETEROSEXUAL. REPENSANDO LA PSICOTERAPIA DE PAREJAS ${ }^{1}$
}

\author{
Juan José Martínez Ibáñez ${ }^{2}$ \\ SEP-IPA, IPR, IARPP, IAN
}

En una época como la actual, donde se está produciendo un cambio de paradigma en la ciencia, el tener que repensar los conceptos anteriores, es una necesidad. El autor, propone una nueva manera de entender el aspecto comunicacional que ocurre dentro de la relación de una pareja heterosexual. Afirma que la misma realidad es percibida de manera diferente dependiendo del funcionamiento mental de cada miembro de la pareja heterosexual. Sugiere que estas dificultades en la comunicación, están provocadas por la diferencia de género. Y son la causa de la mayoría de los conflictos que se dan en la relación de las parejas heterosexuales. Y propone un abordaje, que ayudaría a evitar estas complicaciones.

Palabras clave: Mente, funcionamiento mental, pareja, comunicación, género, neurociencia, relación, emoción.

In a time like the current one, where a paradigm shift in science is taking place, having to re-think the previous concepts is a must. The author proposes a new way of understanding the communicational aspect that occurs within the relationship of a heterosexual couple. He says that the same reality is perceived differently, depending on the mental functioning of each member of the heterosexual couple. He suggests that these difficulties in communication are motivated by gender difference. And they are the cause of most of the conflicts that occur in the relationship of heterosexual couples. And it proposes an approach that would help avoid these complications.

Key Words: Mind, mental functioning, couple, communication, gender, neuroscience, relationship, emotion.

English Title: CONFUSION OF TONGUES IN HETEROSEXUAL COUPLE. RE-THINKING COUPLES

PSYCHOTHERAPY

\section{Cita bibliográfica / Reference citation:}

Martínez Ibáñez, J.J. (2018). Confusión de lenguas en la pareja heterosexual. Repensando la psicoterapia de parejas. Clínica e Investigación Relacional, 12 (1): 106-131. [ISSN 1988-2939]

[Recuperado de www.ceir.info] DOI: 10.21110/19882939.2018.120109

\footnotetext{
${ }^{1}$ Este trabajo ha sido leído como comunicación en las $V$ Jornadas de Psicoanálisis Relacional, organizadas por el Instituto de Psicoterapia Relacional en el Real Sitio de La Granja de San Ildefonso (Segovia), los días 23 y 24 de febrero de 2018.

2 Juan José Martínez Ibáñez es psicólogo clínico, psicoanalista y psicoterapeuta relacional. Es miembro de la Sociedad Española de Psicoanálisis, es miembro invitado de la Sociedad Británica de Psicoanálisis, y miembro de la Asociación Psicoanalítica Internacional. Ha creado y dirige el Centro de Psicoterapia Giralda, en Sevilla, centro asociado del Instituto de Psicoterapia Relacional. También es miembro titular del Instituto de Psicoterapia Relacional, y es vicepresidente del capítulo español de IARPP (International Association for Relational Psychoanalysis and Psychotherapy). También es miembro de IAN (International Attachment Network). Es profesor del Máster en Psicoterapia Relacional/Especialista en Psicoterapia Psicoanalítica Relacional. Ha presentado comunicaciones en diferentes congresos psicoanalíticos internacionales y ha publicado artículos en diferentes revistas de psicoanálisis. La evolución de su pensamiento parte de la corriente principal del psicoanálisis hacia el psicoanálisis relacional, integrando los aportes de Bowlby, Bion, Fonagy, Stern y Siegel, y desarrollando un método terapéutico que se practica en el Centro de Psicoterapia Giralda. Es autor de los libros Las dos edades de la mente: vicisitudes del funcionamiento mental, y El enigma de la angustia: una conceptualización de la angustia desde el vértice relacional.
} 


\section{INTRODUCCIÓN}

"...también me agradaría que a partir de ahora concedieran más importancia a la manera de pensar y de hablar de sus niños, de sus pacientes y de sus alumnos, tras los cuales se ocultan críticas, de forma que pudieran aclarar la confusión de lenguas y aprovecharan la ocasión para aprender cosas." Sandor Ferenczi

Hace unos 86 años, en 1932, Ferenczi escribió un artículo, llamado "Confusión de lenguas entre los adultos y el niño" que, en su contenido, proponía un cambio en la teoría psicoanalítica que Freud estaba desarrollando. Es sabido, que existían grandes diferencias entre lo postulado por Ferenczi y lo afirmado por Freud. Diferencias que acabaron con un distanciamiento entre ambos, cuando en algún momento de su relación, habían sido tan íntimos. Ferenczi comenzó a poner más el acento en la situación traumática real, o sea, en la realidad externa, en el papel de la madre real y en la interacción con esa realidad.

Cuando Ferenczi desarrolla sus ideas en ese artículo, él está afirmando que gran parte de los problemas que existen en la relación entre los padres y sus hijos, es debida, entre otras cosas, a que hay una diferencia en el grado de comprensión de la realidad por parte de ambos. Pero como Freud estaba empeñado en poner en primer lugar a las fantasías, como la estructuradora del psiquismo humano, y, sobre todo, a las fantasías sexuales, hablar de la realidad era, y lo sigue siendo para esa teoría, una herejía.

De modo que Ferenczi se arriesgó demasiado al proponer algo que era tan descabellado para Freud y sus discípulos, y sufrió la humillación de la expulsión y el descrédito del círculo más cercano a Freud. Pero los años han pasado, la ciencia siguió con sus investigaciones, y hoy sabemos que aquellas ideas de Ferenczi, que eran trasnochadas para Freud y el psicoanálisis clásico, eran acertadas. y que, por el contrario, lo postulado por Freud y los psicoanalistas clásicos son una visión errónea del funcionamiento mental humano. Lo que voy a proponer, también puede parecer algo descabellado para algunos colegas, pero tiene como base a los fundamentos científicos avalados por las investigaciones.

El artículo que presento, está relacionado con el funcionamiento mental dentro de la pareja heterosexual. Por un lado, este artículo es un homenaje a Sandor Ferenczi, y, por otro lado, una actualización de sus ideas, aplicadas a la pareja heterosexual, teniendo en cuenta el desarrollo científico actual. Para escribirlo me he basado en las investigaciones científicas actuales y en la experiencia que he tenido, a través de mi práctica profesional con muchas personas que atravesaban dificultades con sus parejas heterosexuales. Algunas conclusiones a las que he llegado son personales, y tendrán que ser avaladas por las investigaciones. 
Espero que, con el tiempo, los resultados de las investigaciones puedan corroborar algunas de mis intuiciones. En caso contrario, me alegraré de haber contribuido a generar inquietud y curiosidad en el ámbito profesional y científico, primer paso para posteriormente poder confirmar una nueva realidad.

Como afirman Isidoro Berenstein y Janine Puget:

"El término "pareja matrimonial", designa una estructura relacional entre dos personas desde un momento dado, cuando establecen el compromiso de formarla en toda su amplitud, lo puedan cumplir o no. La denominación de este objeto de estudio surge del lenguaje convencional y, como siempre, cuando se incorporan denominaciones coloquiales en una teoría, es necesario volver a definir los términos". (p. 13)

Esta afirmación corresponde a un libro titulado "El psicoanálisis de la pareja matrimonial", escrito por ambos autores, en el año 1988, y a los que les guardo un cálido agradecimiento, porque con ellos aprendí a trabajar con parejas. Pero el tiempo ha pasado, la ciencia ha seguido avanzando, y hoy nos encontramos desarrollando un nuevo paradigma científico, como consecuencia de los avances producidos en los distintos campos de la ciencia. Estos autores continúan diciendo que:

"La definición de pareja matrimonial es un requerimiento para ubicar este tipo de relación diádica y diferenciarlo de las relaciones diádicas no matrimoniales". (p. 16)

Más adelante definen que otras relaciones diádicas son los vínculos de amigos y de amantes. Yo le he agregado el término de "pareja de hecho", porque a todos los efectos, su funcionamiento es igual al de la pareja matrimonial, y el término heterosexual, porque es el campo de estudio que he decidido explorar.

El término de pareja matrimonial, lo podemos abordar desde distintos puntos de vista, porque es una estructura multinivel, o sea, que se forma teniendo en cuenta diferentes niveles. Podemos abordarla desde el amor, o desde la teoría del apego, o desde el punto de vista social, o como una estructura de toma de decisiones, o desde la sexualidad, o como una estructura que refleja relaciones de poder, o como una estructura que establece vínculos con las familias de origen de cada miembro de la pareja, etc.

Yo enfocaré este tema de la pareja matrimonial heterosexual desde la perspectiva del funcionamiento mental, porque hoy tenemos constancia científica que hombres y mujeres, funcionamos a nivel mental de distinta manera. Poco a poco nos iremos introduciendo en algunos conceptos actuales aportados por las investigaciones científicas que nos ayudarán a redefinir tanto el concepto de pareja como a la relación que se da entre ambos miembros de la pareja. Para ello tendremos en cuenta algunos aportes que nos vienen de la neurociencia. 


\section{ALGUNOS APORTES DE LA NEUROCIENCIA SOBRE LA INFLUENCIA DEL GÉNERO EN EL CEREBRO EN DESARROLLO}

Actualmente estamos viviendo una época apasionante de cambio de paradigma. La revolución de la neurociencia centrada en los aspectos emocionales, ha revelado la primacía del afecto, de lo emocional, en la condición humana. Y si todo esto lo aplicamos al conocimiento de las similitudes y las diferencias entre el funcionamiento mental de los varones y de las mujeres, sus resultados nos permitirán poder ofrecerles a nuestros pacientes, una comprensión mayor de lo que ocurre cuando se relacionan con sus parejas.

Todos sabemos que los hombres y las mujeres somos distintos. Pero solamente esta afirmación la tenemos en cuenta en lo que respecta a las diferencias anatómicas, y a las diferencias fisiológicas. O sea, a algo físico, material, observable a simple vista. Pero hoy sabemos que el funcionamiento mental de los hombres es distinto al de las mujeres, y viceversa. O sea, que la misma realidad la percibimos de manera distinta. Y, por lo tanto, cada uno intentará adaptarse a esa realidad con su mente.

Pero el vivir compartiendo tiempo, al principio tiempo parcial, cuando la pareja se está conociendo, lo que conocemos como noviazgo, y luego a tiempo completo, a través de una convivencia cuando se van a vivir juntos, obliga a tener que comunicarse, surgiendo entonces problemas de comunicación que lleva a grandes discusiones y enfrentamientos, y a veces a la ruptura, porque cada miembro de la pareja intenta imponer al otro miembro su convicción de que su idea es la que vale y espera que sea aceptada. Esto está basado en la creencia, errónea hasta ahora, de que nuestro funcionamiento mental es el mismo. Dicho de una manera más sencilla de entender, se cree que pensamos de la misma manera.

En lo que sigue, tendré en cuenta los aportes que nos ofrece la Doctora Louann Brizendine en sus libros El cerebro masculino y El cerebro femenino. Estos son dos libros que están muy bien documentados por innumerables investigaciones científicas. Ella nos dice en El cerebro masculino que:

"Algunas áreas y funciones del cerebro están estructuradas de manera diferente en el hombre y en la mujer $y$, con el tiempo, han evolucionado para producir versiones más logradas de hombre y mujeres. Por ejemplo, los circuitos cerebrales que nos alertan del peligro (la amígdala) y nos ayudan a recordarlo (el hipocampo) son las fuentes de las diferencias sexuales e individuales de la memoria emocional. En Hamman (2005), los autores hallaron diferencias sexuales en la respuesta de la amígdala durante las actividades relacionadas con la emoción, como la formación de la memoria emocional y las conductas sexuales. Para ampliar datos sobre la evolución de los circuitos cerebrales masculinos y 
femeninos, véase Linderfors (2007) y Dunbar (2007). Estos autores afirman: "... existen notables diferencias entre los dos sexos en los mecanismos sociales y las unidades cerebrales implicadas. La sociabilidad femenina (que es más afiliativa) se relaciona más estrechamente con el volumen del neocórtex, mientras que la sociabilidad masculina (que es más competitiva y combativa) se relaciona más con las unidades subcorticales (notablemente las asociadas con las respuestas emocionales). Así pues, las diversas unidades cerebrales han respondido a distintas presiones de la selección". (p. 169).

De lo anterior extraemos la idea de que las estructuras cerebrales y la biología hormonal tanto del hombre como de la mujer, generan una realidad exclusivamente masculina o femenina respectivamente. Todas las investigaciones que se vienen realizando en neurociencia, específicamente acerca del funcionamiento del cerebro masculino, arrojan unos resultados sorprendentes. Se sabe que las hormonas y las estructuras cerebrales exclusivas de los varones, crean en todas las fases de la vida, una realidad masculina esencialmente distinta de la femenina, que también suele ser objeto de errores conceptuales y de una excesiva simplificación.

Los cerebros masculinos y femeninos difieren desde el momento de la concepción. Parece una obviedad afirmar que todas las células del cerebro y del cuerpo del hombre son masculinas. Esto significa que existen profundas diferencias en el nivel de todas las células, entre el cerebro masculino y el femenino.

Una célula masculina presenta un cromosoma "Y" inexistente en las células femeninas. Tal diferencia, pequeña pero significativa, tiene su repercusión desde las primeras fases formativas del cerebro, pues los genes sientan las bases de la posterior amplificación que desarrollarán las hormonas. Unas ocho semanas después de la concepción, los diminutos testículos masculinos empiezan a producir la suficiente testosterona para impregnar el cerebro y alterar su estructura de una manera fundamental.

A lo largo de la vida del hombre, el cerebro se formará y reformará según un programa diseñado por los genes y por las hormonas sexuales masculinas. Y esta biología cerebral masculina dará lugar a las conductas característicamente masculinas.

En el cerebro femenino, el estrógeno, la progesterona y la oxitocina son hormonas que predisponen los circuitos cerebrales hacia las conductas típicas femeninas. En el cerebro masculino, son la testosterona, la vasopresina y una hormona llamada SIM (sustancia de inhibición mülleriana) las que causan los efectos más tempranos y duraderos.

La Dra. Brizendine en relación al cerebro femenino nos aclara lo siguiente: 
"Más del 99\% del código genético de los hombres y de las mujeres es exactamente el mismo. Entre 25.000 a 30.000 genes que hay en el genoma humano, la variación de menos del 1\% entre los sexos resulta pequeña, pero esa diferencia de porcentaje influye en cualquier pequeña célula de nuestro cuerpo, desde los nervios que registran placer y sufrimiento, hasta las neuronas que transmiten percepción, pensamientos, sentimientos y emociones". (p.23)

Desde hace tiempo se sabe que los cerebros de las mujeres y de los hombres no son iguales. Los cerebros de los varones son más grandes en alrededor de un $9 \%$, incluso después de la corrección por el tamaño corporal. En el siglo XIX los científicos interpretaron que esa diferencia demostraba que las mujeres tenían menos capacidad mental que los hombres. Lo que hoy sabemos, es que las mujeres y los hombres tienen el mismo número de células cerebrales. Las células están simplemente agrupadas con mayor densidad en las mujeres que en los hombres.

Durante el siglo $\mathrm{XX}$, la mayoría de los científicos creyeron que las mujeres eran esencialmente hombres, limitados neurológicamente y en todos los demás sentidos, excepto en lo relativo a las funciones reproductivas. Esta creencia ha seguido siendo el meollo de los duraderos malentendidos acerca de la psicología y la fisiología femeninas. Hasta la década de los 9o, los investigadores dedicaron poca atención a la fisiología, la neuroanatomía y la psicología femeninas. La escasa investigación de la que se disponía indicaba que las diferencias cerebrales, aunque sutiles, eran profundas.

En todas las mujeres, cuando menstrúan, el cerebro femenino cambia un poco cada día. Algunas partes del mismo cambian hasta el $25 \%$ cada mes. Si la realidad de una mujer podía cambiar radicalmente de semana en semana, lo mismo podría decirse de los cambios hormonales masivos que ocurren a lo largo de la vida de una mujer. A través de innumerables investigaciones sobre este tema, se llega a la conclusión de que el cerebro femenino está tan profundamente afectado por las hormonas que puede decirse que la influencia de éstas, crea una realidad femenina.

A causa de las fluctuaciones que comienzan nada menos que a los tres meses y duran hasta después de la menopausia, la realidad neurológica de una mujer no es tan constante como la de un hombre. Ahora gracias a los avances de la genética y sobre todo a los avances en la tecnología, tenemos la posibilidad de conocer que pasa dentro de nuestro cerebro, en nuestras conexiones neuronales. A través de la tomografía de emisión de positrones (PET) y a la resonancia magnética funcional por imágenes (IRMf) podemos ver lo que ocurre dentro del cerebro humano en tiempo real.

Como resultado, los científicos han documentado una sorprendente colección de diferencias cerebrales estructurales, químicas, genéticas, hormonales y funcionales entre las mujeres y 
los varones. Ahora sabemos que los hombres y las mujeres tienen diferentes sensibilidades cerebrales ante el estrés y el conflicto. Que utilizan diferentes áreas y circuitos cerebrales para resolver los problemas, procesar el lenguaje, y para experimentar y almacenar la misma emoción intensa.

Los cerebros masculinos y femeninos procesan de diferentes maneras los estímulos, como oír, ver, sentir y juzgar lo que otros están sintiendo. Nuestros distintos sistemas cerebrales de operar en el hombre y en la mujer son en su mayoría compatibles y afines, pero realizan y cumplen los mismos objetivos y tareas utilizando circuitos neuronales distintos.

Bajo un microscopio o un examen de IRMf, las diferencias entre los cerebros masculinos y femeninos revelan ser complejas y extensas. En los centros del cerebro para el lenguaje y el oído, las mujeres tienen un $11 \%$ más de neuronas que los hombres. El eje principal de la formación de la emoción y la memoria, el hipocampo, es también mayor en el cerebro femenino, igual que en los circuitos neuronales para el lenguaje y en la observación de las emociones de los demás. Los hombres en cambio, tienen dos veces y media más de espacio cerebral dedicado al impulso sexual, igual que los centros cerebrales más desarrollados para la acción y la agresividad. Estas variaciones estructurales básicas podrían explicar las diferencias en la percepción de la realidad.

Los hombres también tienen procesadores mayores en el núcleo del área más primitiva del cerebro, la amígdala, que registra el miedo y dispara la agresión. El estrés psicológico del conflicto se registra más profundamente en zonas del cerebro femenino. Aunque vivimos en el mundo urbano moderno, habitamos cuerpos hechos para vivir en la naturaleza salvaje, y cada cerebro femenino lleva dentro de él los antiguos circuitos de sus vigorosísimas antepasadas, diseñadas para el éxito genético, pero manteniendo los instintos profundamente instalados que se desarrollaron como respuesta al estrés experimentado en el antiguo mundo salvaje.

Nuestras respuestas al estrés estaban diseñadas para reaccionar ante el peligro físico y situaciones que amenazaran la vida. Ahora esta respuesta al estrés hay que emparejarla con los modernos desafíos de hacer malabarismos con las demandas de la casa, los niños y el trabajo fuera y dentro de casa, sin apoyo suficiente. Por eso tendremos una situación en la cual las mujeres pueden llegar a sentir que unas meras facturas impagadas les generan un estrés que parece amenazar su vida. Esta respuesta impele al cerebro femenino a reaccionar como si la familia estuviera en peligro de sufrir una catástrofe inminente.

Por otro lado, el cerebro masculino no tendrá la misma percepción, a menos que exista amenaza de peligro físico inmediato. Estas variaciones estructurales básicas de sus cerebros 
constituyen el fundamento de muchas diferencias cotidianas en el comportamiento y en las experiencias vitales de los hombres y de las mujeres.

La biología representa el fundamento de nuestras personalidades y de nuestras tendencias de comportamiento. Cuando reconocemos que nuestra biología está influenciada por otros factores, incluyendo a nuestras hormonas sexuales, podemos evitar que el proceso establezca una realidad física que nos gobierna. El cerebro no es nada más que una máquina de aprender dotada de talento. No hay nada que esté absolutamente fijado. Si las sustancias químicas que actúan sobre el cerebro pueden crear realidades diferentes, ¿qué ocurre cuando dos cerebros tienen diferentes estructuras? No cabe duda de que sus realidades serán diferentes.

Se suele opinar que el género fue creado culturalmente para los humanos, pero no para los animales. Hoy se sabe por las investigaciones, que los cerebros macho y hembra de los demás animales, empezaban desarrollándose de modo diferente en el útero, sugiriendo que impulsos tales como el emparejamiento, el embarazo, la crianza de la prole, están plasmados en los circuitos neuronales del animal. Sin embargo, se nos enseña que para los humanos las diferencias sexuales provienen principalmente de que los padres los eduquen como a un niño o como a una niña. Ahora sabemos que esto no es verdad del todo.

Otro autor que me parece muy importante a tener en cuenta, por sus investigaciones recientes en este campo de las diferencias de género, es Allan Schore. En un libro que acaba de salir, de Eva Rass, (2018) sobre la obra de Allan Schore, él nos dice que:

"En las últimas décadas, muchos adultos, probablemente bajo la influencia de la emancipación femenina, han tendido a retirarse de la tarea de asignar un significado prosocial al género, especialmente en lo que respecta a los niños. Sin embargo, ser negligentes con las necesidades de los géneros puede ser peligroso. El riesgo de no tener en cuenta a las diferencias reales que existen entre hombres y mujeres puede tener consecuencias perjudiciales, tanto físicas como psicológicas. Las investigaciones recientes documentan que existe una mayor vulnerabilidad de los hombres al autismo, a un inicio temprano de la esquizofrenia, al trastorno por déficit de atención e hiperactividad (TDAH) y a los trastornos de conducta, así como a un reciente aumento generalizado de estos trastornos en la cultura estadounidense". (Posición 5098 en la versión eBook).

Allan Schore ha sugerido que es el momento adecuado para abordar y explorar los mecanismos más profundos tanto en el desarrollo, como neurobiológicos y culturales, que subyacen a la observación de los niños varones. Las investigaciones muestran claramente que el proceso de maduración cerebral se produce más rápidamente en las niñas que en los niños, un hecho que está respaldado por la evidencia general de su desarrollo físico, 
comportamental y socioemocional. Este mecanismo neurobiológico de desarrollo cerebral más lento en el varón en comparación con los cerebros femeninos se expresa inmediatamente al nacer.

Las diferencias de género en la capacidad de respuesta social se han documentado a las pocas horas de nacer. Los varones recién nacidos responden menos a los estímulos auditivos y sociales y son menos capaces de mantener el contacto visual, y sonríen menos que las mujeres recién nacidas. Ellos también experimentan mayores dificultades para mantener la regulación afectiva que las niñas recién nacidas. Poco después del nacimiento, los niños muestran más irritabilidad, llanto, muecas faciales y labilidad de los estados emocionales. Los varones recién nacidos muestran labilidad de los estados emocionales y también un aumento más rápido de la excitación y un pico de agitación más rápido. Los varoncitos se involucran en menos auto-confort, un comportamiento adaptativo que funciona para regular los períodos de excitación, tensión, excitación o angustia.

Schore presenta estudios que indican que la maduración prenatal y postnatal de la excitación autónoma (Sistema Nervioso Autónomo) y el Sistema Nervioso Central de los varones es más lenta que en el desarrollo femenino. Estas diferencias de género se centran en las diferencias en las tasas de desarrollo social y emocional, comenzando en los primeros estadios de la vida. Debido a su mayor reactividad emocional y a los retrasos en la autorregulación de sus estados afectivos, los niños necesitan confiar más en las aportaciones reguladoras maternas que las niñas y, por lo tanto, son más demandantes del apoyo social del sujeto de apoyo primario, que generalmente es la madre. Las niñas, que maduran más rápidamente, son menos vulnerables al estrés interactivo normal. Estas diferencias de género en la expresividad y en la autorregulación emocional, afectan diferencialmente las demandas reguladoras de madres e hijos, así como de madres e hijas. De hecho, durante la infancia, los varones muestran una inmadurez cerebral estructural prolongada en el procesamiento afectivo y en los circuitos cerebrales de regulación del estrés.

Schore propuso que el hemisferio derecho, dominante para procesar y regular la emoción, se desarrolla más lentamente en los bebés varones que en las bebés mujeres. La maduración cerebral sería más rápida en las niñas, al igual que el desarrollo físico y psicológico, de modo que la inmadurez prolongada de los niños representaría un riesgo para un período de tiempo más largo. De hecho, la investigación ahora documenta que, comenzando en el útero, los cerebros de hombres y mujeres muestran una sensibilidad diferencial hacia el estrés de la vida temprana.

Además de ser más sensibles a los estresores sociales normales, los varones también son más sensibles a los estresores sociales extremos. Schore citó estudios que muestran que el 
cortisol materno alto al final del embarazo se asocia con un comportamiento más difícil durante las semanas 1-7 posnatales. Estos bebés muestran más expresiones faciales negativas y de llanto, una descripción que se ha denominado "un temperamento difícil". La idea sostenida durante mucho tiempo, que describía el temperamento fijo "innato" que representa factores genéticos que se expresan primero al nacer es incorrecta. El temperamento "biológico" al nacer es más bien el resultado de mecanismos epigenéticos que han evolucionado prenatalmente, y continúan siendo epigenéticamente modelados o deformados por el entorno social y emocional después de nacer.

Además, en los estadios postnatales del desarrollo y debido a un período prolongado de inmadurez límbica, los hombres son más susceptibles que las mujeres al "trauma relacional", especialmente al abuso y a la negligencia tempranos. Estas formas más severas de estrés en la vida temprana se reflejan en las diferencias de género documentadas en la traumatología del desarrollo y en los niveles más frecuentes de apegos inseguros y en apegos desorganizados de alto riesgo en los infantes tanto en hombres como en mujeres. Por lo tanto, Schore afirmó que, debido al retraso en las tasas de maduración cerebral en los bebés, las diferencias de género deben ser tenidas en cuanta durante el desarrollo emocional y especialmente en la traumatología del desarrollo, que a su vez son factores de alto riesgo para los trastornos psiquiátricos que se forman posteriormente.

En general, la tesis central del trabajo de Schore afirma que se observan diferencias de género significativas entre hombres y mujeres en las funciones sociales y emocionales en los estadios más tempranos del desarrollo, y que éstas son consecuencias no solo de las diferencias en las hormonas sexuales y en las experiencias sociales, sino también de las tasas de maduración cerebral masculina y femenina, específicamente en el cerebro derecho durante el desarrollo temprano.

Los circuitos de regulación del estrés del cerebro masculino se desarrollan más lentamente que los de la mujer en los períodos críticos prenatal, perinatal y postnatal, y esta maduración estructural diferencial se refleja en las diferencias de género normales en las funciones de apego del cerebro derecho. Debido a este retraso en la maduración, los varones en desarrollo también son más vulnerables durante un período largo de tiempo a los factores estresantes en el entorno social (trauma de apego) y a las toxinas en el ambiente físico (interruptores endocrinos) que afectan negativamente el desarrollo del cerebro derecho.

En términos de diferencias en la psicopatología relacionada con el género, los mecanismos neuroendocrinológicos y neurobiológicos del desarrollo temprano que están involucrados en la mayor vulnerabilidad de los varones al autismo, al inicio temprano de la esquizofrenia, al TDAH y a los trastornos de conducta, también como a los mecanismos epigenéticos, pueden expresa. Este material es para uso científico y profesional exclusivamente y puede contener información clínica sensible. Los editores no se responsabilizan de los contenidos de los autores. Dirigir las consultas sobre derechos y autorizaciones a ceir@psicoterapiarelacional.es 
explicar el reciente aumento en la propagación de estos trastornos en la cultura estadounidense y en otras culturas.

Schore sobre la investigación de género sugiere que, debido a una flexibilidad y adaptabilidad emocional más avanzada, las niñas, que comienzan en preescolar y en el jardín de infantes, muestran más habilidades para hacer frente a los estresores sociales propios de los contextos educativos. Por lo tanto, se está ampliando la brecha educativa entre niñas y niños, especialmente en circunstancias económicas menos favorables.

Debido al aumento en los varones de las dificultades durante el desarrollo, a los trastornos de conducta y a los trastornos de aprendizaje provocados por el TDAH, muchos niños de todas las culturas tienen dificultades educativas y están rezagados con respecto a las tasas de graduación tanto en la escuela secundaria como en la universidad. De hecho, en muchos países, los niños tienen más probabilidades que las niñas de repetir un año escolar o de recibir educación especial.

Por lo tanto, la educación por parte de los maestros de preescolar y de jardín de infantes con respecto a las diferencias sociales y emocionales entre niños y niñas es una necesidad absoluta. Las familias deberían ser informadas sobre la neurobiología del apego y sobre el hecho de que la necesidad del bebé varón de una regulación temprana del afecto, no puede ser satisfecha algunas semanas después del nacimiento, por más que se le llame cuidado temprano, que ahora es muy común en muchas culturas occidentales.

Las políticas escolares deben pensar en la dinámica del apego emocional en la relación profesor-alumno en el aula, y no solo sobre estimular a las mentes de manera cognitiva. Sin un cambio en estas perspectivas, lo más probable es que los varoncitos continúen quedándose atrás. A diferencia de la emancipación femenina que ha permitido cada vez más que en muchos países las mujeres alcancen objetivos profesionales, el hecho de que los hombres no adquieran las mismas competencias educativas no solo daña su vida en general, sino que también presenta simultáneamente situaciones problemáticas para las mujeres (Rass, 2011a, 2011b).

Como hemos visto a través de estos aportes de Lou Brizendine y de Allan Schore, que, a su vez, remiten a muchas investigaciones que se han hecho sobre el tema de las diferencias en el desarrollo en los hombres y en las mujeres, podemos comprobar que la idea sostenida en nuestra educación de que somos iguales es un error, y en algunos casos, un error grave. De modo que, si esto lo aplicamos a la relación entre un hombre y una mujer, en un contexto específico como es el de la pareja, nos va a ayudar a comprender cómo podemos 
relacionarnos mejor, aceptando estas diferencias. Pero para ello, primero tenemos que conocer a la mente y al funcionamiento mental masculino y femenino.

\section{LA MENTE Y EL FUNCIONAMIENTO MENTAL}

Para poder entender que el funcionamiento mental masculino es diferente del femenino, primero tenemos que conocer a la mente. Es muy llamativo, y a veces, hasta desconcertante, que todos los que nos dedicamos a la salud mental, no tengamos una definición de la mente. El poder tenerla, nos podría permitir, por ejemplo, poder saber con cierta exactitud, qué nos pasa a los seres humanos a nivel mental.

La definición de la mente, como he dicho anteriormente, está ausente en los campos científicos que tratan sobre la vida mental. Por eso algunas veces la palabra mente, se usa como una señal de algo desconocido, otras veces como una señal de una fuente misteriosa de nuestra vida interna subjetiva, otras veces se la equipara al cerebro. Pero creo que es muy importante, dar el paso de explorar una definición de la mente, para poder lograr una comprensión, de lo que puede ser realmente una mente sana. No debemos olvidarnos que los seres humanos, somos lo que nuestra mente quiere que seamos. De modo que cuanto más la conozcamos, mejores serán los recursos mentales que podremos utilizar, y, por lo tanto, mejor será nuestra calidad de vida.

¿Por qué no tenemos una definición de la mente? ¿De donde viene esta idea tan extendida entre los seres humanos de que no hace falta definirla? Todos los seres humanos partimos de la idea de que tenemos una mente, y además con algunas pequeñas diferencias, que tenemos la misma mente. Tantos hombres como mujeres. Esta es una consecuencia de creer que la mente es una herencia filogenética. Si la mente es algo que tenemos todos y además es la misma para todos, se entiende que no haya hecho falta definirla. Lo que hoy sabemos, gracias a las investigaciones que se han hecho en los distintos campos de la ciencia, es que heredamos de manera filogenética a través de los procesos evolutivos, la arquitectura biológica, los procesos neurobiológicos, la disponibilidad para crear una mente.

Pero no heredamos una mente, sino que la mente se construye, se crea, o se co-crea como veremos un poco más adelante. De modo que, con la educación actual, se refuerza la idea de que la mente es una "cosa" que tenemos todos y, por lo tanto, no hace falta definir a esa "cosa" que tenemos todos. Lo que si ha habido son intentos de describirla, como hizo Freud cuando elabora la primera tópica y la segunda tópica o estructural del aparato psíquico. Otra descripción que perdura todavía es la que dice que la mente es el cerebro. Pero hasta ahora no ha habido una definición de la mente. 
No debemos olvidarnos que los seres humanos somos nuestra mente. Nuestra mente es la que gobierna nuestros actos, por lo tanto, es la que manda en nuestras vidas. Si no la conocemos, ella nos lleva a donde quiera llevarnos. Por eso es muy importante conocerla. Porque si la conocemos, podremos llevar a nuestra mente a donde nosotros queramos.

Ya hemos visto cómo las recientes investigaciones desde un conjunto de disciplinas científicas, nos aportan unos conocimientos que nos permiten tener una mejor comprensión del funcionamiento mental humano. Las distintas disciplinas científicas, nos dan muchos puntos de vista de cómo la mente funciona, aportándonos conocimientos en profundidad, y desde distintas perspectivas de la experiencia humana.

Yo propondré, teniendo en cuenta algunos aportes de Daniel Siegel, $(2016,2017)$, una definición de la mente, que nos permitirá poder entendernos a todos. Dice dicho autor que la mente es un proceso (que no es estática, que siempre está cambiando y yo agrego, que este proceso es siempre cognitivo y afectivo), que emerge de nuestro cuerpo (cerebro y sistema nervioso) y de las relaciones con las demás personas que están en nuestro entorno más cercano. Y que además tiene una función, que es la de regular el flujo de energía y de información.

De modo que un aspecto central de la mente, es que procesa de manera relacional, y corporal, el flujo de energía y de información. El flujo de energía y de información, es lo que compartimos las personas dentro de una cultura, y este flujo, es lo que podemos medir en los sujetos, a través de un scanner. Para decirlo de manera más simple, las conexiones mentales humanas, a través de las experiencias cognitivas y afectivas reales intersubjetivas íntimas, modelan las conexiones nerviosas, y esta relación, contribuye a la creación de la mente de cada uno.

Las relaciones interpersonales íntimas, y las redes neuronales juntas, modelan la mente. La mente es más que la suma de las partes; es la esencia de lo emergente. Tenemos que aceptar que la mente, es algo más que una actividad que realiza el cerebro. La neurociencia actual, como hemos visto anteriormente, revela la conexión entre las estructuras del cerebro, y su función, y nos da nuevos aportes, sobre cómo la experiencia real, modela los procesos mentales.

Nosotros ahora sabemos, que la experiencia real, y la activación (firing) de las neuronas, pueden alterar a las moléculas reguladoras, que controlan la expresión de los genes, que constituyen un proceso llamado epigénesis. Estos cambios epigenéticos, revelan las poderosas formas a través de las cuales, la experiencia real, modifica la manera en la que el cerebro se desarrolla, a lo largo de la vida. 
Además, los recientes estudios en neuroplasticidad del cerebro, han dado como resultado que el cerebro, continúa modificando sus conexiones estructurales con la experiencia real, a través de toda la vida. Los últimos estudios nos dicen, que nuestro cerebro mamífero es profundamente social, y que las relaciones tienen un enorme impacto sobre las funciones neuronales, desde los tempranos días de nuestra vida.

La mente, -los procesos reguladores cognitivos y afectivos, que crean patrones en el flujo de energía y de información- puede ser descrita, como emanando en parte, pero sólo en parte, desde la actividad de las neuronas. Los seres humanos nos hemos desarrollado para ser seres sociales, y los procesos mentales son un producto, tanto de nuestras conexiones neuronales internas, como de las conexiones interpersonales con los otros significativos. Si no tenemos esto en cuenta, nos resultará fácil deslizarnos hacia el modo lineal de pensar, creyendo que la mente es solamente la actividad de nuestro cerebro.

Como vemos, la mente sería un proceso de regulación (para mí la regulación es siempre, cognitiva y afectiva), del flujo de energía y de información y que se llevaría a cabo a través de las relaciones interpersonales íntimas a través de un proceso comunicacional y procesadas a nivel cerebral por las conexiones neuronales que dejarían huellas en nuestro cerebro. Me parece acertada esta definición, porque refleja el funcionamiento mental humano actual.

Hoy sabemos que la mente es un proceso auto-organizado, que emerge de las propiedades de un sistema. Este proceso surge de la interacción de los elementos del sistema (flujo de energía y de información dentro del cuerpo y que son distribuidos entre las personas), y también regula de una manera recursiva, los elementos de los cuales surgió. Esto significa que las relaciones y el cerebro modelan a la mente y la mente modela las relaciones y el cerebro. La mente, el cerebro y las relaciones son tres aspectos de un sistema: regulación, mecanismo encarnado y distribución de flujo de energía y de información.

Aprender acerca del aspecto regulador de la mente, nos capacita para ver más profundamente, las formas en que la mente se desarrolla, ver los cambios en el cerebro, y ver cómo las relaciones evolucionan con el tiempo. Cuando estos elementos actúan, generan como hemos dicho anteriormente, huellas en el cerebro. Esas huellas son también conocidas como los Modelos Operativos Internos, un aporte de John Bowlby (1969) o como yo la defino, como la Parte Infantil de la Mente (Martínez Ibáñez, 2013). Estos modelos, o, dicho de otro modo, la mente, el cerebro y las relaciones, pueden ser intencionalmente movidas hacia la salud. Estos modelos de vida saludable, implican la integración de la energía, y de la información, dentro del sistema nervioso, y entre las personas. La integración, es el principio organizador, que vincula la forma en que el flujo de energía, y de información, es compartido 
(relaciones), es modelado por los mecanismos del sistema nervioso encarnado (embodied) o dicho simplemente, el cerebro, y es regulado (la mente).

Creo que llegar a definir, que un aspecto central de la mente, es que procesa a nivel cognitivo y afectivo, de manera relacional, y corporal, el flujo de energía y de información, es un paso importante. Hoy sabemos que necesitamos de los otros significativos, que nuestra mente se desarrolla, gracias a nuestro encuentro con alguien, que cuidará de nosotros. Esas relaciones dejarán huellas en nuestro funcionamiento mental, que no se borrará nunca. Esas huellas nos acompañarán el resto de nuestra vida, hasta el último día. Ellas constituirán nuestros modelos mentales inconscientes relacionales. Inconscientes, porque se generan, cuando aún no tenemos lenguaje para comunicarnos y relacionales porque se crean en una relación. Ese es nuestro inconsciente relacional.

Por lo tanto, a nivel mental nosotros funcionamos con los contenidos que tenemos, no funcionamos con los que no tenemos. Cuesta creerlo, porque nos han educado en la creencia errónea de que nuestra mente es algo especial, superior, y que gracias a ella nos hemos convertido en la especie que está en la cúspide de la evolución. Pero esto no es así, nuestra mente es el resultado de una co-construcción con otra mente de alguien significativo.

El saber que sólo funcionamos mentalmente con los contenidos que tenemos en nuestra mente, nos permitirá ser más empáticos, más solidarios, más cooperativos, con los demás. Quizás si conocemos un poco mejor nuestro funcionamiento mental, podamos mejorar como especie. Esta aventura de vivir, hoy sabemos que dependerá, de con quién nos encontremos al salir del cuerpo de nuestra madre.

Nuestra mente se origina en una relación de apego. Por lo tanto, poder saber qué tipo de relación de apego estableceremos con nuestra madre, determinará el desarrollo de nuestra mente. Por eso es tan importante, tener en cuenta, la trascendencia de ese momento. $Y$ de todos los demás momentos, que nos toquen vivir. Si las futuras madres, junto con el entorno que tienen a su alrededor, pueden tener en cuenta, que cuidar mentalmente, cognitiva y afectivamente, de sus hijos, tanto cuando son pequeños como a medida que se van haciendo adultos, es el mejor legado que le podrán dar, seguramente podremos conseguir que la vida en este mundo, sea menos difícil, estresante, angustiante y violenta de lo que es hoy. De cómo se lleve a cabo esa relación de apego incipiente que comienza a desarrollarse desde el nacimiento, dependerá el funcionamiento mental que tendremos.

Ya hemos definido a nuestra mente, a la mente de los seres humanos, pero ahora también sabemos que la mente masculina es diferente de la mente femenina, y viceversa. De modo que ahora tendremos que articular estos conocimientos diferenciados del funcionamiento mental con respecto al género, con el funcionamiento mental dentro de una pareja 
heterosexual. Aunque tendremos que definir qué es una pareja matrimonial o de hecho heterosexual.

\section{QUÉ ES UNA PAREJA, MATRIMONIAL O DE HECHO, HETEROSEXUAL}

Ante todo, tenemos que tener presente que los hombres y las mujeres son animales. Esta es una afirmación que nos escuece por dentro, porque tener que aceptarla, es como tener que asumir algo que nos hace bajar del pedestal, donde nos hemos colocado. Nos cuesta mucho aceptar esta afirmación, pero creo que tenemos que desarrollar esta idea. Para ello voy a hacer un esquema según Linneo. Recordemos que Linneo fue el creador de la clasificación de los seres vivos. Y también nos dio un lugar en esa clasificación.

\begin{tabular}{|l|l|}
\hline SUPERREINO & EUKARYOTA \\
\hline REINO & ANIMALIA \\
\hline SUBREINO & EUMETAZOA \\
\hline SUPERFILO & DEUTEROSTOMIA \\
\hline FILO & CHORDATA \\
\hline SUBFILO & VERTEBRATA \\
\hline INFRAFILO & GNASTHOSTOMATA \\
\hline SUPERCLASE & TETRAPODA \\
\hline CLASE & MAMMALIA \\
\hline SUBCLASE & THERIA \\
\hline INFRACLASE & PLACENTALIA \\
\hline SUPERORDEN & EUARCHONTOGLIRES \\
\hline GRANORDEN & EUARCHONTA \\
\hline ORDEN & PRIMATES \\
\hline SUBORDEN & HAPLORRHINI \\
\hline INFRAORDEN & SIMIIFORMES \\
\hline PARVORDEN & CATARRHINI \\
\hline (SIN RANGO) & CRANIATA \\
\hline SUPERFAMILIA & HOMINOIDEA \\
\hline FAMILIA & HOMINIDAE \\
\hline SUBFAMILIA & HOMININAE \\
\hline TRIBU & HOMININI \\
\hline SUBTRIBU & HOMININA \\
\hline GÉNERO & HOMO \\
\hline ESPECIE & HOMO SAPIENS \\
\hline & \\
\hline
\end{tabular}


Es frecuente que nos cueste aceptar algunas de estas clasificaciones, por ejemplo, que somos una tribu o que pertenecemos al orden de los primates. Parece que eso es algo primitivo, pero, aunque nos cueste aceptarlo, lo somos. ¿Por qué es importante aceptar esto? Porque los hombres y las mujeres de nuestra especie, tenemos que desarrollar funciones como especie. Es cierto que hemos evolucionado mucho desde nuestra prehistoria, pero también es cierto que seguimos siendo macho y hembra. Y esto obliga a tener que desarrollar distintas funciones para que la especie se siga manteniendo.

Por ejemplo, nuestros ancestros machos, tenían la función de cuidar el territorio y proveer alimento. En aquellos tiempos había predadores que les perseguían y había que sobrevivir. Es cierto que, en nuestros tiempos, los únicos predadores que hay, están dentro de nuestra especie y son los psicópatas. Por lo que ahora no hay que preocuparse tanto como en la prehistoria. Pero los rasgos se siguen manteniendo, aunque con diferencias, como resultado de la evolución. Ahora los machos humanos son más sofisticados que antes, pero algunas características se siguen manteniendo.

Actualmente, es muy frecuente que nos lleguen a nuestras consultas, quejas, lamentos y a veces hasta enfados, debido a que los varones no hablan dentro de una relación de pareja. Las mujeres lo expresan como quejas, y los varones como culpa. Pero nos vendría bien saber, que esta es una actitud, que va más allá de nuestra voluntad.

Para poder sobrevivir en la época en la que había predadores, era muy importante no delatarse, no hacer ruido, o sea, no hablar o hablar lo mínimo. Ya hemos dicho que la función del macho de la especie era cuidar el territorio, por lo tanto, no hablar, era una forma de cuidar a la pareja, a la familia y a la descendencia. Los machos de la especie, por más que quieran y lo intenten no pueden modificar este rasgo ancestral, porque está en juego la supervivencia de la especie. Si esta característica, la podemos comprender como un rasgo epigenético evolutivo, provocará menos discusiones entre los miembros de una pareja heterosexual cuando se enfrenten a unas situaciones similares

Ahora vamos a hablar de algunas funciones de la hembra de la especie. La principal función de la hembra de la especie homo sapiens, como de tantos mamíferos, es tener crías y ocuparse de la crianza de ellas. Para poder conseguirlo con éxito, las hembras vienen preparadas con un repertorio de comportamientos que permiten, que se perpetúe la especie. Uno de ellos, por ejemplo, es ser muy exigentes, muy perfeccionistas, en relación a lograr la supervivencia de las crías. Inmediatamente después de nacer su cría, surge una preocupación desmedida con la labor que tienen que desarrollar como madres. Los mecanismos mentales anticipatorios, en relación a los cuidados que tendrán que dar a sus hijos, se incrementan y provocan vivencias de sufrimiento. En cuanto el bebé llora, ya están preguntándose cuál 
puede ser la causa que provoca ese malestar. En cuanto ven en los ojos de sus hijos algo diferente a lo habitual, ya están alarmándose pensando en algo malo.

Esas actitudes permiten asegurar la supervivencia de sus hijos, y por lo tanto de la especie. $Y$ esto lo tienen que compartir con sus parejas varones, que no tienen desarrollado este aspecto, provocando discusiones y a veces hasta desprecio, porque se les dice que son exageradas. Para que un bebé sobreviva, hace falta que alguien repare en algún rasgo del bebé o del niño, para que se le pueda dar una solución. De modo que, no es verdad que las mujeres sean exageradas, sino que los varones, al no tener este aspecto desarrollado, creen que esa preocupación es desproporcionada. Y esta situación, muy común en la pareja como padres, provoca muchas discusiones y enfados, que se podrían aliviar si se supiera que son inevitables porque somos psicológicamente distintos.

Aclarado lo anterior, que me parece fundamental para poder entender por qué surgirán problemas en las parejas en general, vamos a definir a la pareja.

En realidad, el concepto "pareja" define algo que es co-creado entre dos personas. No hay dos parejas iguales, lo mismo que no hay dos personas iguales. El término pareja define un proceso. Al igual que cuando hablamos de la definición de mente, y dijimos que es un proceso, el concepto de pareja, también es un proceso, siempre está cambiando. Siempre está en evolución.

Una vez que ya hemos definido, de manera amplia, qué es una pareja, vamos a centrarnos en el tipo de pareja específica sobre la que quiero que conozcamos más. Me gustaría que pudiéramos definir qué es una pareja matrimonial o de hecho heterosexual. Parece que todos estaríamos de acuerdo en lo que es, porque estamos familiarizados desde pequeñitos con esta idea. Pero creo que hace falta definirla, para aportar claridad a esta Torre de Babel en la que hemos convertido a la humanidad, donde creemos que sabemos de lo que hablamos. Para mí, la pareja matrimonial, o, de hecho, heterosexual como tal, como una cosa, no existe. A partir de ahora, para no repetir constantemente esta denominación, diré solamente la $\mathrm{PMHH}$.

La PMHH es el resultado de la unión legal o religiosa de dos personas de distinto sexo, por eso el nombre de pareja, un par, con el objetivo de llevar adelante sus vidas dentro de un proyecto compartido. Si tenemos en cuenta que estamos hablando de dos personas de distinto sexo, lo importante será que cada una de ellas, mantenga su individualidad. Y si decimos que tendrán un proyecto compartido, lo importante será que ambos integrantes de la $\mathrm{PMHH}$, vayan redefiniendo en cada momento, cuáles serán esos proyectos que tendrán que compartir. 
Por lo tanto, la pareja es un proceso. Siempre está en movimiento, siempre está cambiando. No deberíamos convertirla en una cosa, en algo estático, porque en ese momento, empezarán los problemas. Y, por otro lado, si hablamos de dos individuos, tenemos inevitablemente que hablar de dos mentes, porque las personas somos nuestra mente. $Y$ como hemos definido anteriormente, la mente, el funcionamiento mental, también es un proceso. Nosotros, los seres humanos, siempre estamos naciendo a una nueva realidad. Ya sea a nivel individual, de pareja, familiar o social.

¿Por qué se nos hace difícil entender que la pareja es un proceso, que siempre está cambiando? Porque desde que nacemos, nuestra mente se va desarrollando con otra mente, que es la que nos va dando los modelos mentales que luego desarrollaremos a lo largo de nuestra vida. Y vamos aprendiendo que hay que vivir en pareja para sentirse bien. Como algo que viene dado, no como algo que hay que construir, que hay que crear.

Y si a lo anterior, le agregamos que cuando más fuerza tiene esta idea de la pareja es en la adolescencia, se hace más comprensible que sea algo mecánico que llevamos adelante. Porque la adolescencia, tal como está planteada actualmente en nuestro desarrollo, es una etapa de una inseguridad máxima, de una fragilidad alarmante y de un desvalimiento total. Se entiende que cada adolescente busque desesperadamente a alguien que le aporte lo que le falta para poder sentirse bien. Y generalmente esa seguridad aparece a través de la pareja de novios. Los y las adolescentes, se sienten más protegidos o protegidas si tienen a alguien a su lado. De modo que va se va haciendo efectiva la idea infantil de que hay que tener una pareja para poder sentirse bien.

En mi libro Las dos edades de la mente, planteo que, en nuestro desarrollo mental, todos los modelos operativos internos que nuestra mente ha ido recibiendo a lo largo de nuestro desarrollo, forman parte de nuestro inconsciente relacional y yo lo denomino la parte infantil de la mente. Esa parte infantil de la mente es la que predomina en nosotros y en relación a la $\mathrm{PMHH}$ es la que impulsa a la búsqueda de alguien que complemente, de manera satisfactoria, esas carencias provocadas por la fragilidad, la inseguridad y el desvalimiento que sentimos. Esta es la causa de que luego usemos el tópico de la media naranja, como manera de entender la $\mathrm{PMHH}$.

Cuando creemos en el mito de la media naranja, creemos en la perfección de encontrar a alguien que está hecho para estar con nosotros, creemos en una relación donde todo encaja. Y muchas veces pasa que, cuando conocemos a alguien, parece que es perfecto para nosotros, empezamos una relación sorprendidos por lo bien que nos hemos acoplado, ¡estamos hechos el uno para el otro! 
El que la humanidad, o sea nosotros, hayamos creado el mito de la media naranja, es debido a que, en nuestro desarrollo mental, sobre todo a partir de la adolescencia, no nos dan las herramientas para poder crecer con seguridad, y con confianza en nosotros mismos. Por lo tanto, se hace imprescindible que alguien nos complemente, nos de lo que nos falta, para poder ser felices. Pero aquí es donde comienzan los problemas, porque ese otro o esa otra, también espera lo mismo, y cuando no se consigue lo que se espera del otro o de la otra, la frustración hace su aparición.

Por lo que sería recomendable que, en vez de propagar el mito de la media naranja, nos esforcemos por desarrollar la idea de que para tener una buena PMHH sería mejor contar con dos naranjas. Es muy importante modificar esta idea, para poder tener una sociedad más saludable mentalmente, y más satisfactoria socialmente. Las dos naranjas serían cada miembro de la $\mathrm{PMHH}$. Sé que esta idea pueda parecer inapropiada cuando hablamos de una $\mathrm{PMHH}$. Pero esto no es más que una consecuencia de los modelos mentales que nos dieron desde pequeños en relación a lo que debe ser una pareja.

Aunque parezca paradójico, para que una $\mathrm{PMHH}$ funcione mejor, será conveniente, que cada uno se esfuerce por poder ser feliz de manera individual. Que logre sentirse bien de manera personal, consigo mismo. Para que después esa felicidad, ese bienestar, cuando llegue a su casa, lo pueda compartir con su pareja. Hoy sabemos que las emociones son contagiosas. De modo que, si uno de los dos miembros de la PMHH llega a su casa sintiéndose bien, podrá compartir ese bienestar con su pareja. Podrá contagiar emocionalmente a su pareja con su felicidad. Lo mismo pasa si uno de los miembros de la PMHH llega triste o infeliz, también va a contagiar emocionalmente a su pareja. Por lo tanto, el funcionamiento de una $\mathrm{PMHH}$ dependerá del estado de ánimo que tenga cada miembro de la $\mathrm{PMHH}$.

Al creer, erróneamente, que éramos iguales, se ha establecido un funcionamiento, en el que se espera que cada miembro de la $\mathrm{PMHH}$, pueda comprender al otro miembro de la $\mathrm{PMHH}$, casi de manera mágica. Y cuando esto no ocurre, aparece en el funcionamiento de la $\mathrm{PMHH}$ una frustración. Y si esto se repite con frecuencia, el desgaste comienza a hacer su aparición y a veces, termina con la ruptura de la pareja.

Si sabemos, como ahora se sabe, que ambos miembros de la $\mathrm{PMHH}$ funcionan de manera diferente, podremos conseguir que los momentos en los que se pueda compartir algo entre ellos, sea más agradable, más armónico, más saludable. Y, además, esto lo podemos conseguir, tanto si tratamos a los miembros de la pareja de manera conjunta, como a cada miembro de la pareja de manera individual. A continuación, a través de una viñeta clínica, podremos entender mejor estas ideas. expresa. Este material es para uso científico y profesional exclusivamente y puede contener información clínica sensible. Los editores no se responsabilizan de los contenidos de los autores. Dirigir las consultas sobre derechos y autorizaciones a ceir@psicoterapiarelacional.es 


\section{VIÑETA CLÍNICA}

Cierto día se presenta en la consulta, una persona de alrededor de 70 años, con un aspecto de abatimiento. Entra arrastrando los pies, un tanto desaliñado en su manera de vestir, y sentí compasión. Me transmitía, sin hablar, que estaba derrotado. Cuando le pregunto, una vez que toma asiento, ¿qué está pasando?, me dice, casi sin ganas de hablar, que no puede más. Y se mete la mano en el bolsillo de su pantalón y saca un papel y me dice que lo mire. Era una nota del psiquiatra al que había acudido en primer lugar, porque no se sentía bien. La nota decía que esta persona padecía de una depresión reactiva a una situación ambiental. La leo en voz alta y le pregunto si está de acuerdo con lo que dice la nota, y me dice que sí y que no puede más.

Me cuenta que lleva cinco años soportando humillaciones, insultos, y desprecio por parte de su mujer, y que ya no puede más, y dice que, para vivir así, mejor morirse. Le pregunto por qué dice que desde hace cinco años le pasa esto. $Y$ comenta que hace cinco años su mujer tuvo una operación importante, una histerectomía, y desde entonces está peor con él. Pero que con anterioridad también ella actuaba de esta manera, aunque de manera menos intensa. "Las descalificaciones, los insultos, los desprecios, ahora son continuos, y ya no puedo más", dice mirando hacia abajo y con desconsuelo.

Esta descripción es bastante frecuente en los relatos de los pacientes, con respecto a sus relaciones de pareja, sean hombres o mujeres. ¿Por qué pasa esto en las relaciones de pareja? ¿Por qué son frecuentes las discusiones?, ¿Por qué, en general, son por cosas intranscendentes? ¿Qué es lo que lleva a ambos miembros de una pareja a tener que discutir acaloradamente, insultar, despreciar, minusvalorar, de manera mutua? ¿Y por qué después de tanta agresividad verbal, y a veces hasta física, se llega a la reconciliación?

Esto es algo que desde que empecé a trabajar con parejas me ha llamado la atención. Esta necesidad de defender posturas a rajatablas, aún a riesgo de poner en peligro la estabilidad emocional de la pareja, por motivos que no son los que llevaron a la creación de la pareja, como pueden ser los temas de la vida cotidiana de una pareja, los horarios, las responsabilidades en cuanto a las tareas, el orden, la limpieza, los momentos de espera, etc. son, los que me han movido a seguir investigando sobre este tema.

A través de los aportes actuales que nos llegan de la neurociencia, de la antropología, de la biología, y de otras disciplinas científicas, he llegado a la conclusión de que esto sucede por lo que he comentado anteriormente, acerca de que el funcionamiento mental de cada miembro de la $\mathrm{PMHH}$ es distinto, es diferente. Los hombres y las mujeres perciben la misma realidad de manera diferente. El mismo hecho, es percibido de manera distinta. Y por supuesto que ninguno de ambos, tienen la verdad absoluta sobre ese hecho. Es verdad que 
cada uno sostendrá que su punto de vista es el correcto, y lo hará de manera intransigente. Pero lo que hoy vamos sabiendo es que tenemos percepciones diferentes sobre el mismo hecho.

Cuando este paciente, me cuenta su sufrimiento cuando recibe los desprecios y los insultos por parte de su pareja, yo lo estoy escuchando desde esta nueva manera de entender la relación de pareja. Y comienzo a preguntarle si él cree que su mujer no lo quiere. Y me dice que a veces tiene dudas sobre esto, pero que en el fondo le quiere.

Esta afirmación la he comprobado en la mayoría de los y las pacientes. ¿Por qué es importante esta pregunta? Porque permite que el paciente se escuche a sí mismo. Si su mujer le quiere, los motivos por los que ella se expresa de esta manera serán otros. Una vez que el paciente reconoce que su mujer le quiere, entonces le sigo preguntando por qué cree que lo hará. Y no tiene respuesta. Esto también es algo frecuente en los pacientes. Se quedan sin respuesta. Entonces le invito a que podamos empezar a pensar, a mentalizar, a reflexionar sobre esto y sobre el funcionamiento mental.

Es muy importante que los pacientes conozcan a su mente. Porque, lamentablemente no se conoce. $Y$ esto se debe a que no se la ha definido hasta ahora. El poder tener una definición de la mente, nos permite acercarnos con más confianza, y con más seguridad, a la hora de poder ayudarles a los pacientes y a las personas en general, a que puedan saber por qué se genera su sufrimiento.

Una vez que van comprendiendo que la mente tiene dos partes, una que conecta con la realidad (la parte realista de la mente) y otra que funciona como cuando éramos pequeños (la parte infantil de la mente), y que además puedan entender que el esfuerzo por conseguir las cosas reales, es la capacidad que nos diferencia a los niños de los mayores, en cuanto al contacto con la realidad, y que la angustia es una emoción, es cuando están preparados para saber que hombres y mujeres funcionamos de manera distinta.

Y comienzo a explicarle que hombres y mujeres somos distintos, no solamente anatómicamente, y fisiológicamente, sino también psicológicamente. Y le sigo explicando que las mujeres funcionan mentalmente, de manera distinta a los varones y viceversa. $Y$ le aclaro, que ni mejor ni peor, sino distinta. Que percibimos la misma realidad de manera diferente. $Y$ que, por lo tanto, valdrá la pena detenernos a conocer algunas de esas diferencias.

A partir de este momento, le explico algunas de las diferencias entre el funcionamiento mental masculino y el femenino, para que le sirvan a la hora de tener que hacer el esfuerzo de conectar mejor con sus parejas mujeres y viceversa. Y como lo que le trae a la consulta, es 
un sufrimiento intenso acerca de los insultos, las descalificaciones y la agresividad verbal por parte de su mujer, le empiezo a explicar algunas características sobre este tipo de funcionamiento mental respecto a las mujeres.

Una de ellas, y esto tiene que ver con lo que le pasa a este paciente, es que la mujer piensa en voz alta. Esto es lo contrario que los varones, que piensan en silencio. Para comprender esto tenemos que volver a recordar que somos animales, que somos mamíferos que pertenecemos al reino animal, y como tales, venimos preparados para cumplir funciones diferentes. Los varones son los machos de la especie y las mujeres son las hembras de la especie. Los machos, vienen preparados para cuidar el territorio de posibles predadores y de proveer alimentos, y las hembras vienen preparadas para tener crías y cuidar de ellas.

Por supuesto que los Homo Sapiens hemos evolucionado y en el siglo XXI hemos sofisticado mucho nuestras características iniciales, pero hay funciones que no podemos modificar sustancialmente. Por poner algunos ejemplos, todavía los machos de la especie no podemos tener hijos, y las hembras de la especie no han desarrollado una musculatura que le permita realizar grandes esfuerzos físicos. De modo que las hormonas, como hemos visto anteriormente, nos condicionan a desarrollar actividades diferentes y en lo posible, complementarias.

Voy a poner un ejemplo, que todos los podemos comprobar. En una pareja, con la que se comparten algunos momentos, es muy frecuente que las mujeres digan en voz alta: "dónde están las llaves", o "dónde está el móvil". Todo esto ocurre mientras están buscando dentro de sus bolsos interminables las llaves o el móvil. Esto lo hacen siempre. El varón que está cerca, escucha esas palabras y, por ejemplo, podría hacer algún comentario descalificatorio hacia su mujer, o también podría intentar ayudar. Vamos a pensar sobre este último caso, el de querer ayudar. Esto lo hace con su mejor buena voluntad, y usando su mente masculina, dice algo parecido a esto: "por qué no pones las llaves o el móvil en uno de los bolsillos internos que tiene el bolso". Esto lo dice, porque como ya hemos aclarado anteriormente, los varones tienen que dar soluciones para que todo funcione bien. $Y$ la mujer cuando escucha esto, comienza a decirle algo parecido a esto: "Ya sé que, para ti, todo lo que hago, lo hago mal" o dice "me quieres dejar, ya sé que eres perfecto". Todo lo anterior lo dice enfadada y provoca que el varón que ha querido ayudar le diga, algo parecido a esto: "bueno, yo sólo quería ayudar", y la mujer suele contestar algo parecido a esto: "ayudar, ...lo que quieres es criticarme, decirme que todo lo hago mal, cuando vas a decirme que hago algo bien", y a esto le sigue una discusión acalorada que deja a ambos molestos.

Esto que he comentado es un ejemplo, pero en la realidad algo similar sucede con mucha frecuencia en las relaciones de pareja. Y ocurre porque funcionamos mentalmente de 
manera diferente. Antes hemos dicho que percibimos la misma realidad de manera diferente. Ante un mismo hecho, cada uno lo evalúa de manera diferente. El hecho que acabo de exponer, el de que la mujer busque las llaves y lo diga en voz alta, es el mismo hecho tanto para el varón como para la mujer. Entonces, ¿por qué pasa esto? Porque las mujeres cuando piensan en voz alta, están evacuando una tensión, un estado de angustia. La angustia que les está generando que no encuentran las llaves o el móvil en este caso (pero que esto es extensivo a cualquier situación que les genera tensión). Los varones, por su parte, cuando hablan, lo hacen solamente para comunicarse con los demás. Si no, no lo hacen, se quedan callados. Hablar por hablar no es una práctica de los varones. Pero en las mujeres, el hablar no solamente es para comunicar, sino que también lo usan para aliviar tensiones. Este uso del lenguaje hablado es distinto. Ni mejor ni peor.

Las mujeres cuando quieren hablar para comunicar algo importante, suelen empezar la frase con alguna palabra cariñosa que cada pareja tiene establecida, como "cariño", "amor", "gordo", "papi", etc., y luego comentan el tema que les interesa. De modo que los varones deberían aprender que no tienen que escuchar a sus mujeres todo el tiempo, sino solamente cuando usen esas palabras cariñosas. De esta manera se ahorrarán ambos integrantes de la pareja muchas discusiones estériles. Digo estériles, porque esto no tiene fin, porque la mujer no puede dejar de expresarse de esa manera, porque esa es una de sus maneras de aliviar tensiones. Y por su parte, el varón tampoco podrá modificar su tendencia a que todo funcione bien. De modo que será mejor conocer estas diferencias para que la convivencia sea más agradable. Aceptar que somos diferentes nos permitirá vivir mejor en pareja.

El paciente al volver a la siguiente sesión, después de poner en práctica estas ideas, dice que le va mejor. Que no contesta cada vez que su mujer se pone a decirle todas las barbaridades que dice. Y ha comprobado que lo que yo le había dicho le funciona. Que después actúa como si nada, y me dice: "por ejemplo, el otro día, llegué del supermercado, y se me olvidó comprar la lejía, y empezó a insultarme, y yo me acordé de ti, y me quedé callado. Al rato, me dice, ¿te parece que vayamos a comer unas tapitas? Y se me vino tu imagen, cuando me lo decías, y no me lo podía creer. Era verdad, en otros momentos, yo me habría enfadado con ella, habría discutido acaloradamente, y después habría seguido enfadado".

Soy consciente que, en una relación de pareja, influyen muchos factores, pero me he detenido a poder repensar sobre este tema de la comunicación en la $\mathrm{PMHH}$, porque es el que predomina en las relaciones entre los seres humanos en general. Y como psicoterapeuta, mi objetivo es que los pacientes puedan conocer cómo funciona su mente. Que conozcan su propio funcionamiento mental. $\mathrm{Y}$ en una $\mathrm{PMHH}$, conocer el funcionamiento mental, tanto masculino como femenino, es de trascendental importancia a la hora de ayudar a ambos 
miembros de la misma. Y este aspecto de la comunicación en la relación de pareja se puede conocer, tanto si están presentes ambos miembros de la pareja, como si el planteamiento es por parte de uno de los miembros, durante un tratamiento psicoterapéutico individual.

\section{REFERENCIAS}

Bradshaw, G. A., \& Schore, A. N. (2007). How elephants are opening doors: Developmental neuroethology, attachment and social context. Ethology, 113, 426-436.

Bradshaw, G. A., Schore, A. N., Brown, J. L., Poole, J. H., \& Moss, C. J. (2005). Elephant breakdown. Nature, 433, 807.

Brisch, K.-H. (2007). Bindung und Umgang. In Deutscher Familiengerichtstag (Ed.), Siebzehnter Deutscher Familiengerichtstag vom 12-15. September in Brühl/Brühler Schriften zum Familienrecht, Vol. 15, 89-135.

Bielefeld: Gieseking. (En Rass, F.: (2018) The Allan Schore Reader: Setting the Course of Development. Routledge. New York).

Brizendine, L. (2007). El cerebro femenino. RBA Libros. Barcelona.

Brizendine, L. (2010). El cerebro masculino. Las claves científicas de cómo piensan y actúan los hombres y los niños. RBA Libros. Barcelona.

Ferenczi, S. (1933). Confusión de lenguas entre los adultos y el niño. En Obras Completas, Psicoanálisis, Tomo IV, Capítulo IX. Editorial Espasa Calpe. Madrid, 1984.

Martínez Ibáñez, J.J. (2013). Las dos edades de la mente. Vicisitudes del funcionamiento mental. Madrid: Editorial Ágora Relacional.

Puget, J. y Berenstein, I. (1988). Psicoanálisis de la pareja matrimonial. Paidós. Buenos Aires.

Rass, E. (2011a). Erziehung heute. Jungen im Abseits-Mädchen im Erfolg. In Gerd-Bodo von Carlsburg (Ed.), Baltische Stu-dien zur Erziehungs-und Sozialwissenschaft, Bd. 22, 173-191. Frankfurt: Peter Lang. (En Rass, F.: (2018) The Allan Schore Reader: Setting the Course of Development. Routledge. New York).

Rass, E. (2011b). Bindung und Sicherheit im Lebenslauf. Psychodynamische Entwicklungspsychologie. Stuttgart: Klett-Cotta. (En Rass, F.: (2018) The Allan Schore Reader: Setting the Course of Development. Routledge. New York).

Rass, E. (2018). The Allan Schore Reader: Setting the Course of Development. Routledge. New York.

Schore, A. N. (2012). The science of the art of psychotherapy. New York/London: Norton.

Schore, A. N. (2017). All our sons: The neurobiology and neuroendocrinology of boys at risk. Infant Mental Health Journal, 38, 15-52.

Schore, A. N., \& McIntosh, J. (2011). Family law and the neuroscience of attachment, Part 1. Family Court Review, 49, 501-512.

Schore, J., \& Schore, A. N. (2008). Modern attachment theory: The central role of affect regulation in development and treatment. Clinical Social Work, 36, 9-20. 
Siegel, D. (2017). Viaje al centro de la mente. Lo que significa ser humano. Barcelona: Paidos

Siegel, D. (2016). Guía de bolsillo de Neurobiología Interpersonal. Un manual integrativo de la mente. Barcelona: Editorial Eleftheria.

Original recibido con fecha: 25/1/2018 Revisado: 15/2/2018 Aceptado: 28/02/2018 